\title{
CORRECTION
}

\section{Correction: A critical evaluation of current definitions of necrotizing enterocolitis}

Shiloh R. Lueschow, Timothy J. Boly, Elizabeth Jasper, Ravi M. Patel and Steven J. McElroy (D)

(c) The Author(s), under exclusive licence to the International Pediatric Research Foundation, Inc 2021

Pediatric Research (2022) 91:711; https://doi.org/10.1038/s41390-021-01735-9

Correction to: Pediatric Research https://doi.org/10.1038/s41390-

021-01570-y, published online 21 May 2021

In this article the wrong figure appeared as Fig. 2; the figure should have appeared as shown below. The original article has been corrected.

A

\begin{tabular}{|c|c|c|c|c|c|c|}
\hline & KNN & SNN & NB & RF & SVM & DT \\
\hline ST & 1 & Ibfgs solver & Multinomial & 50 estimators & Gamma $=2$ & Max depth $=2$ \\
\hline UK & 3 & 2 layers & Multinomial & $\begin{array}{l}500 \text { estimators } \\
5 \text { max features }\end{array}$ & Gamma $=2$ & Max depth $=3$ \\
\hline $20 f 3$ & 3 & $\begin{array}{l}\text { Ibfgs solver } \\
3000 \text { maxit }\end{array}$ & Multinomial & $\begin{array}{l}500 \text { estimators } \\
5 \text { max features }\end{array}$ & Gamma $=5$ & $\begin{array}{c}\text { Max depth }=4 \\
\text { Min leaf }=5\end{array}$ \\
\hline INC & 1 & $\begin{array}{l}\text { Ibfgs solver } \\
2 \text { layers } \\
4000 \text { maxit }\end{array}$ & Multinomial & $\begin{array}{l}100 \text { estimators } \\
5 \text { max features }\end{array}$ & Gamma $=2$ & $\begin{array}{c}\text { Max depth }=2 \\
\text { Min leaf }=5\end{array}$ \\
\hline VON & 3 & 2 layers & Multinomial & 100 estimators & Gamma = scale & $\begin{array}{c}\text { Max depth }=2 \\
\text { Min leaf }=5\end{array}$ \\
\hline
\end{tabular}

B

\begin{tabular}{|c|c|c|c|c|c|c|}
\hline & KNN & SNN & NB & RF & SVM & DT \\
\hline Bell 2 & 3 & Ibfgs solver & Multinomial & 50 estimators & Gamma $=2$ & Max depth $=2$ \\
\hline Bell 3 & 2 & 1000 maxit & Multinomial & $\begin{array}{l}500 \text { estimators } \\
4 \text { max features }\end{array}$ & Gamma $=2$ & Max depth $=2$ \\
\hline ModBell 2A & 3 & 1400 maxit & Gaussian & $\begin{array}{l}500 \text { estimators } \\
5 \text { max features }\end{array}$ & Gamma $=$ scale & Max depth $=2$ \\
\hline ModBell 2B & 1 & $\begin{array}{l}\text { Ibfgs solver } \\
1000 \text { maxit }\end{array}$ & Multinomial & 500 estimators & Gamma $=3$ & Max depth $=2$ \\
\hline ModBell 3A & 3 & 2 layers & Multinomial & 100 estimators & Gamma = scale & Max depth $=2$ \\
\hline ModBell 3B & 3 & 2 layers & Multinomial & 500 esimators & Gamma $=$ scale & $\begin{array}{c}\text { Max depth }=2 \\
\text { Min leaf }=3\end{array}$ \\
\hline
\end{tabular}

